\title{
Estimation of Aircraft Engine Mount Dynamic Forces based on Least- Squares Scheme
}

\author{
Junwei Xu ${ }^{1+}$, Zixin Feng ${ }^{1}$, Feng $\operatorname{Han}^{1}$, Huayong Zhao ${ }^{1}$ and Chenxi $\mathrm{Li}^{1}$ \\ ${ }^{1}$ Functional Structure Department, Shanghai Aircraft Design and Research Institute, Shanghai, China, \\ 201210
}

\begin{abstract}
In the engineering of noise and vibration, one of the most important things is to determine the dynamic forces caused by engine vibration at the excitation locations on the engine mount. With the determined dynamic forces, the structure dynamic behavior and aircraft cabin noise caused by engine vibration can be predicted and corresponding methods can be used to resolve possible problems. However, it is impossible to measure the dynamic forces at the engine mount caused by engine vibration directly when aircraft is in flight condition. In this paper, an indirect method called the least-squares scheme is implemented to back-calculate the engine mount dynamic forces with the measured frequency response function (FRF) matrices on the ground and measured structural acceleration in the flight condition. The norm condition number is applied to evaluate the inversion process and it shows that these identified dynamic forces on the engine mount meet the requirement of engineering.
\end{abstract}

Keywords: Dynamic forces identification, Least-squares scheme, FRF, Aircraft engine mount.

\section{Introduction}

Aircraft engine mount is a very important structure which attaches engine to the fuselage through pylon. The engine mount not only transfers the engine thrust to the aircraft, but also transfers the vibration caused by the imbalance of engine to the airframe so that the structure-borne noise appears in passenger cabin[1]. For the noise and vibration engineering, one of the most important things is to acquire the dynamic force on the engine mount. With the identified dynamic force, the dynamic behaviour of structures and the structureborne noise caused by engine vibration can be calculated. Accurate identification of dynamic forces can be very important to the design process[2]. The dynamic forces caused by engine operation can be measured when engines are installed on the test bench with special force gauges. But unfortunately, when engines are installed on the aircraft, it is almost impossible to insert force gauges into the force transfer path to measure those dynamic forces directly [3,4]. Therefore, inferring these dynamic forces by using measured structural vibration response in some sort of inverse model is sometimes necessary. Among the conventional force identification methods, the frequency response function (FRF)-based least-squares approach is the most widely used because it can be applied to a variety of force identification problems [5]. Given the measured vibration response at one or more locations and the frequency-domain FRF matrix, the dynamic excitation forces can be back-calculated at each specific frequency by pre-multiplying the measured response vector by pseudo-inverse of the FRF matrix at that frequency. The pseudo-inverse technique is also known as a leastsquares method $[3,4]$.

In 1979, Bartlett [6] applied the least-squares scheme to determine vibratory vertical and lateral hub forces in an experimental helicopter model. The reasonableness of the forces identified in that effort testifies to the success of the method. N. Okubo also successfully identified dynamic forces by applying this approach on various structures [7]. In spite of some successful applications, the accuracy of this approach

\footnotetext{
${ }^{+}$Corresponding author. Tel.: + 86-153 1618 9139; fax: n/a.
}

E-mail address: xujunwei@comac.cc 
can still be hindered by the direct inversion of an ill-conditioned FRF matrix at frequencies near the structural resonances [3,4]. Fabunmi [8,9] found that because only one mode dominates the FRF matrix near resonances for a lightly damped structure, the FRF matrix tends to be ill-conditioned and least squares scheme is most likely to fail at these frequencies and the ill-conditioned matrix can be numerically unsolvable or numerically unstable with regard to error in the response [10]. In the mathematics area, two enhanced filters which are described using a regularization parameter are developed to increase the inverse stability [11]. One of these filters is the truncated singular value decomposition (TSVD) and another one is the Tikhonov filer. The two filters are compared with in many different ways and these contents of comparison are beyond the scope of this paper, and therefore will not be discussed in this paper. However, it was demonstrated that the two enhanced filters schemes are only necessary near the structural resonances $[3,4]$. In the engineering, a criterion which is called norm condition number is applied to determine whether to use the enhanced filters or not.

The following section provides an introduction of least-squares scheme, this is the most widely used for dynamic forces identification. Then a practical application case about the aircraft engine mount dynamic forces identification is stated in details and two different FRF matrices are used to identify the dynamic forces on the engine mount. Comparing with the two norm condition number of the rectangular FRF matrices, it is obviously found that one of the two FRFs is more accurate than the other one. Therefore, the relative errors of the dynamic forces identified by two different FRF matrices are calculated.

\section{Theory for Force Identification}

\subsection{Force identification based on least-square scheme}

The least-square scheme is the most widely used method to for dynamic forces identification. For a vibration structure, the translational response $y(x, t)$ at the $x$ point on a structure relates to the excitation force $f\left(x_{0}, t\right)$ located at $x_{0}$, the time-domain vibration can be described as integral form[5]

$$
y(x, t)=\int_{0}^{t} A\left(x \mid x_{0}, t-\tau\right) f\left(x_{0}, \tau\right) d \tau
$$

Applying the Fourier transform to Eq. (1) and it can be expressed in the frequency domain as

$$
\tilde{y}(x, \omega)=\tilde{A}\left(x \mid x_{0}, \omega\right) \cdot \tilde{f}\left(x_{0}, \omega\right)
$$

where $\tilde{f}, \tilde{y}$, and $\tilde{A}$ are the complex functions of the harmonic frequency $\omega$.

If it belongs to the multiple degrees of freedom vibration system, Eq. (2) can be described in matrix form

$$
\tilde{\boldsymbol{y}}(\omega)_{m \times 1}=\tilde{\boldsymbol{A}}(\omega) \cdot \tilde{\boldsymbol{f}}(\omega)_{n \times 1}
$$

where $m$ denotes the number of measurement locations, and $n$ denotes the number of excitation forces to be identified. $\widetilde{\boldsymbol{A}}(\omega)$ is the FRF matrices with $A_{i j}(\omega)$ being the frequency response function between the measured response at $x_{i}$ and the excitation force at the location of $x_{j}$.For the dynamic forces identification, the $\tilde{\boldsymbol{y}}(\omega)$ and $\widetilde{\boldsymbol{A}}(\omega)$ are known, and the Eq. (3) can be pre-multiplied by $\widetilde{\boldsymbol{A}}^{-1}(\omega)$ to compute the excitation dynamic force $\tilde{\boldsymbol{f}}(\omega)$ if $m=n$. But, in most cases, $m$ does not absolutely equal to $n$, on the other hand, to reduce the impact of errors in the measured response signals, it is better to use a least-squares scheme with $m>n$ when back-calculating the dynamic excitation forces [3]. The least-squares scheme provides the redundancy by using response measurements taken at extra locations to reduce the measured error; therefore this scheme improves the accuracy of the identified forces [3]. The identified forces can be obtained from Eq. (3) as follows

$$
\tilde{\boldsymbol{f}}=\tilde{\boldsymbol{A}}^{+} \cdot \tilde{\boldsymbol{y}}
$$

where $\tilde{\boldsymbol{f}}$ is short for $\tilde{\boldsymbol{f}}(\omega), \tilde{\boldsymbol{A}}$ is short for $\tilde{\boldsymbol{A}}(\omega)$ and $\tilde{\boldsymbol{y}}$ is short for $\tilde{\boldsymbol{y}}(\omega)$. $\tilde{\boldsymbol{A}}^{+}$denotes the pseudo-inverse of the non-square matrix $\widetilde{\boldsymbol{A}}$, which can be defined as [5]

$$
\tilde{\boldsymbol{A}}^{+}=\left(\tilde{\boldsymbol{A}}^{H} \tilde{\boldsymbol{A}}\right)^{-1} \tilde{\boldsymbol{A}}^{H}
$$

where the $H$ superscript denotes the Hermitian transpose. 
The measured structural FRF matrices are the inherent property of structures and they are not related to the excitation forces. In general, the FRF matrices can be obtained by hammer or shaker method. Once the measured structural responses and FRF matrices are obtained, the dynamic forces can be computed from Eq. (4). However, the accuracy of identified forces depends on the accuracy of the matrix inversion process. Unfortunately, the matrices trend to be ill-conditioned especially near the frequencies of structural resonances so that the error in the measured responses $\tilde{\boldsymbol{y}}$ will be amplified and the accuracy of identified dynamic forces will be reduced dramatically. The following section provides a quantitative judgement criterion which is called norm condition number to judge the inverse calculation. The lower value of the norm condition number is, the better identified dynamic forces are acquired.

\subsection{Norm condition number criterion}

Considering the error in the measurement of the structural responses can described by vector $\tilde{\boldsymbol{\eta}}$, so the 'polluted' structural responses can be written as

$$
\tilde{\boldsymbol{y}}_{\text {pol }}=\tilde{\boldsymbol{A}} \tilde{\boldsymbol{f}}_{\text {exact }}+\tilde{\eta}
$$

where $\tilde{\boldsymbol{f}}_{\text {exact }}$ denotes the exact dynamic forces (for example the actual excitation force), and $\tilde{\boldsymbol{y}}_{\text {pol }}$ is the 'polluted' responses containing the error $\tilde{\boldsymbol{\eta}}$. According to the singular value decomposition (SVD)[12], the FRF matrices $\tilde{\boldsymbol{A}}$ can be decomposed by the factorization form

$$
\tilde{\boldsymbol{A}}=\boldsymbol{U} \cdot \boldsymbol{\Sigma} \cdot \boldsymbol{V}^{H}
$$

where $\boldsymbol{U}$ and $\boldsymbol{V}$ are the unitary matrices, $\boldsymbol{V}^{H}$ is the adjoin matrix of $\boldsymbol{V}$ and $\Sigma$ is a diagonal matrix whose elements are referred as the singular values of the matrix $\tilde{\boldsymbol{A}}$.the diagonal matrix $\Sigma$ can be written as

$$
\Sigma=\left[\begin{array}{c}
\operatorname{diag}\left(s_{1}, \ldots, s_{n}\right) \\
{[0]}
\end{array}\right]
$$

where $s_{1}, s_{2} \ldots, s_{n}$ are non-negative singular values arranged in descending order, $\operatorname{diag}\left(s_{1}, \ldots, s_{n}\right)$ is a diagonal matrix with the elements in parentheses located on the main diagonal. With the error expression, Eq. (4) can be written as follows

$$
\tilde{\boldsymbol{f}}_{\text {pol }}=\tilde{\boldsymbol{f}}_{\text {exact }}+\sum_{i=1}^{n} s_{i}^{-1}\left(\boldsymbol{u}_{i}^{H} \eta_{i}\right) \boldsymbol{v}_{i}
$$

where $\boldsymbol{u}_{\boldsymbol{i}}$ is the column vector of $\boldsymbol{U}, \boldsymbol{v}_{i}$ is the $\underset{\tilde{\boldsymbol{A}}}{i}$-th column vector of $\boldsymbol{V}, \eta_{i}$ is the $i$-th element of $\tilde{\boldsymbol{\eta}}, s_{i}$ is the $i-t h$ singular values of matrix $\tilde{\boldsymbol{A}}$. If $\tilde{\boldsymbol{A}}$ is ill-conditioned at some resonant frequencies, the ratio between the largest and smallest singular values tend to be high and the division by the smallest singular values $s_{n}, s_{n-1} \cdots$ in Eq. (9) and the error will be amplified to the identified dynamic forces $[3,4]$. The way to evaluate whether the identified dynamic forces is better or bad is through a threshold criterion. This criterion uses the second norm condition number of the rectangular FRF $\tilde{\boldsymbol{A}}$. This norm, which has been found have a direct relationship with the error amplification effect in the inverse process [13], is defined as the ratio of the largest singular value to the smallest singular value

$$
\kappa(\tilde{\boldsymbol{A}})=\frac{s_{1}}{s_{n}}
$$

where $\kappa(\tilde{\boldsymbol{A}})$ refers to the matrix condition number. If $\tilde{\boldsymbol{A}}$ is ill-conditioned at some frequencies, then $\kappa(\tilde{\boldsymbol{A}})$ will be high. If the norm condition number at the frequency of interest is larger than a threshold value of $\kappa_{t h}$, the enhanced filters, such as TSVD or Tikhonov filter-based least squares schemes will be applied to overcome any inversion singularity. The norm condition number indicates the good for the inverse calculation, the lower value of the norm condition number is, the better identified dynamic forces are. In engineering, a good criterion for the inversion of the matrix is to have a norm condition number lower than 100. In this paper, the aircraft engine mount dynamic forces identification application has a low norm condition number because the author designed the FRFs test and vibration response reasonability. Due to the space limitation of this paper, the enhanced filters theory will not be introduced here, but readers interested in these filters can refer to the resources. 


\section{FRFs of Aircraft Engine Mount and Pylon}

In general, the FRF matrices can be measured by the hammer or shaker method[14]. For a multiple degrees of freedom system, the FRF matrices can be described as

$$
\tilde{\boldsymbol{A}}(\omega)=\frac{\boldsymbol{G}_{f x}(\omega)}{\boldsymbol{G}_{f f}(\omega)}
$$

where $\boldsymbol{G}_{f f}(\omega)$ denotes the auto-power spectrum matrix of excitation force ; $\boldsymbol{G}_{f x}(\omega)$ denotes the crossspectrum matrix between the excitation force and response caused by the force at location $x$. Now, a variety of FFT-based digital spectrum analyzers have the standard test template for the FRF matrices test and calculation based on the input force signals and response acceleration signals. In this paper, the FRF matrices between engine mount and pylon are measured by hammer method. Fig. 1 shows the excitation and response measured locations on the engine mount and pylon.

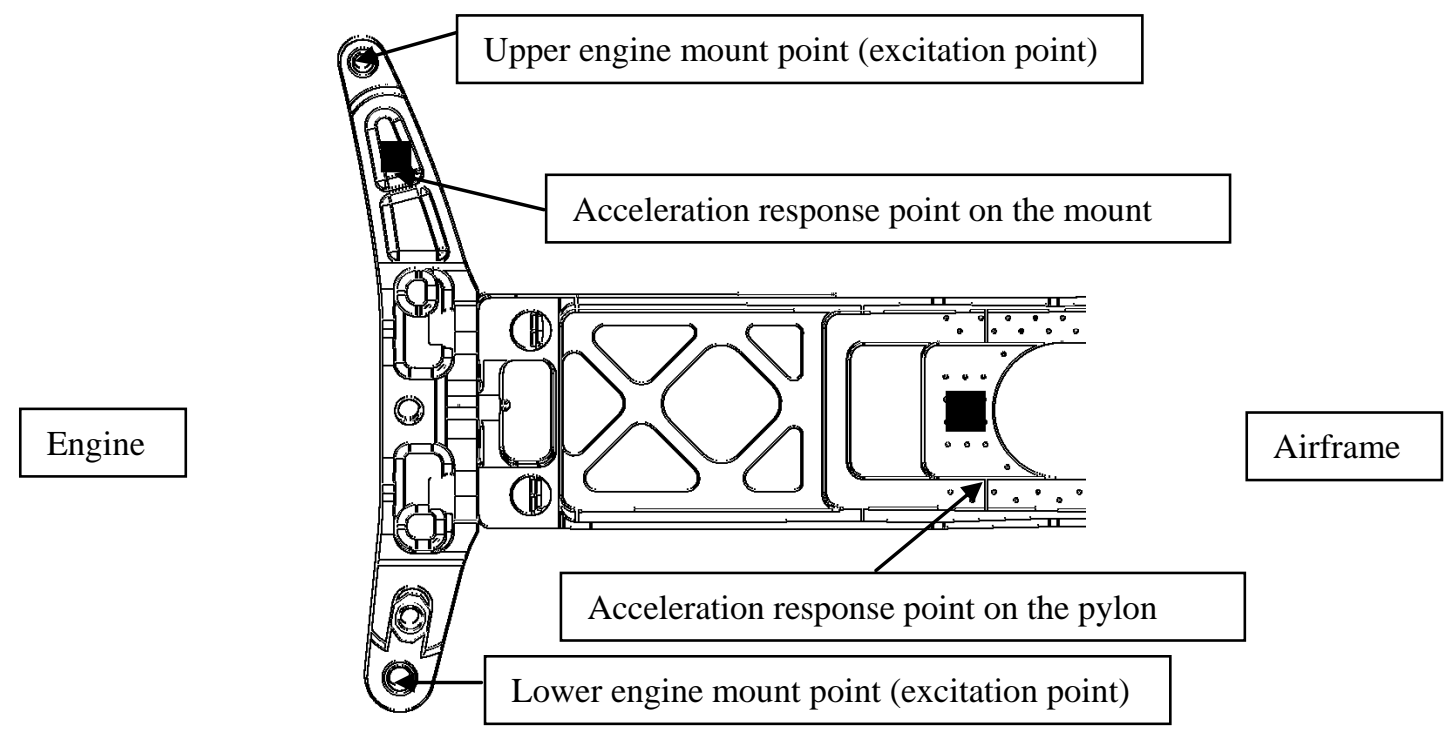

Fig. 1: Engine mount and pylon structures sketch.

In this paper, two groups of FRF matrices were measured by hammer method. The first one group is the FRF matrices between lower/upper excitation points and acceleration response point at the pylon, the second group is the FRF matrices between lower/upper excitation points and acceleration response point on the mount. The FRF matrices test was done when the aircraft parked in hangar. Fig. 2 to Fig. 7 show the measured FRF results of them. In the following section, the two groups of FRF matrices will be used to identify the engine mount dynamic forces on the lower/upper excitation points.
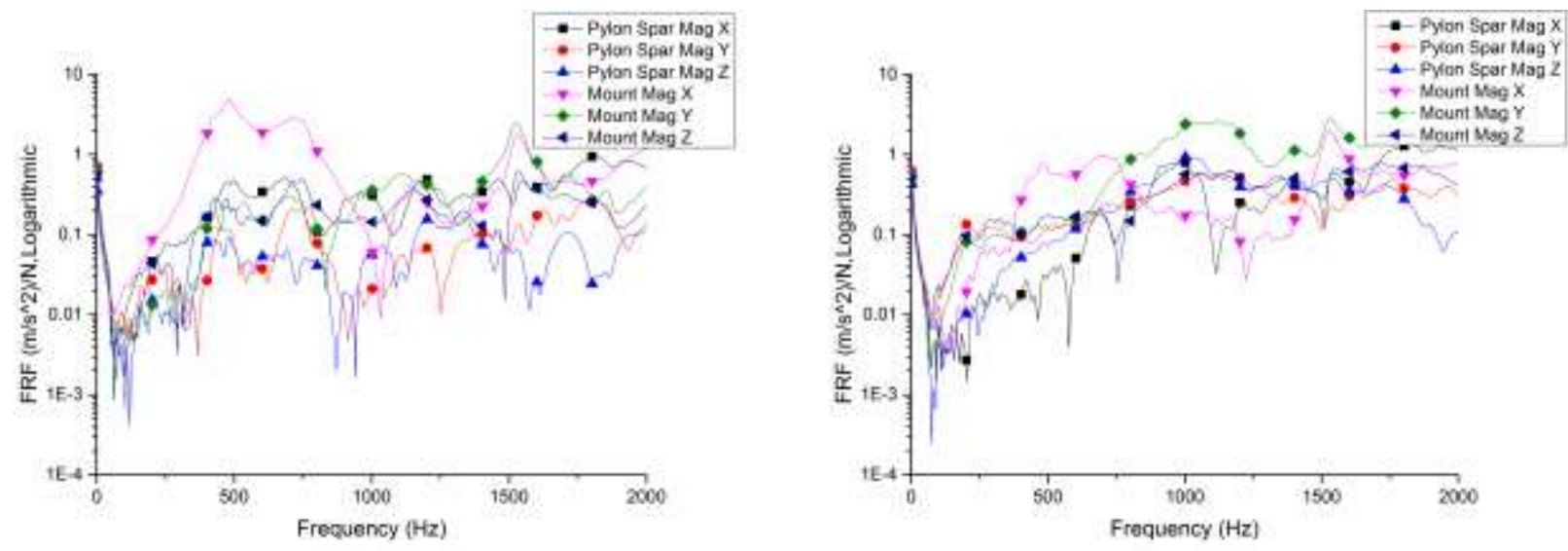

Fig. 2: FRFs of lower excitation point at $\mathrm{X}$ direction. Fig. 3: FRFs of lower excitation point at $\mathrm{Y}$ direction. 

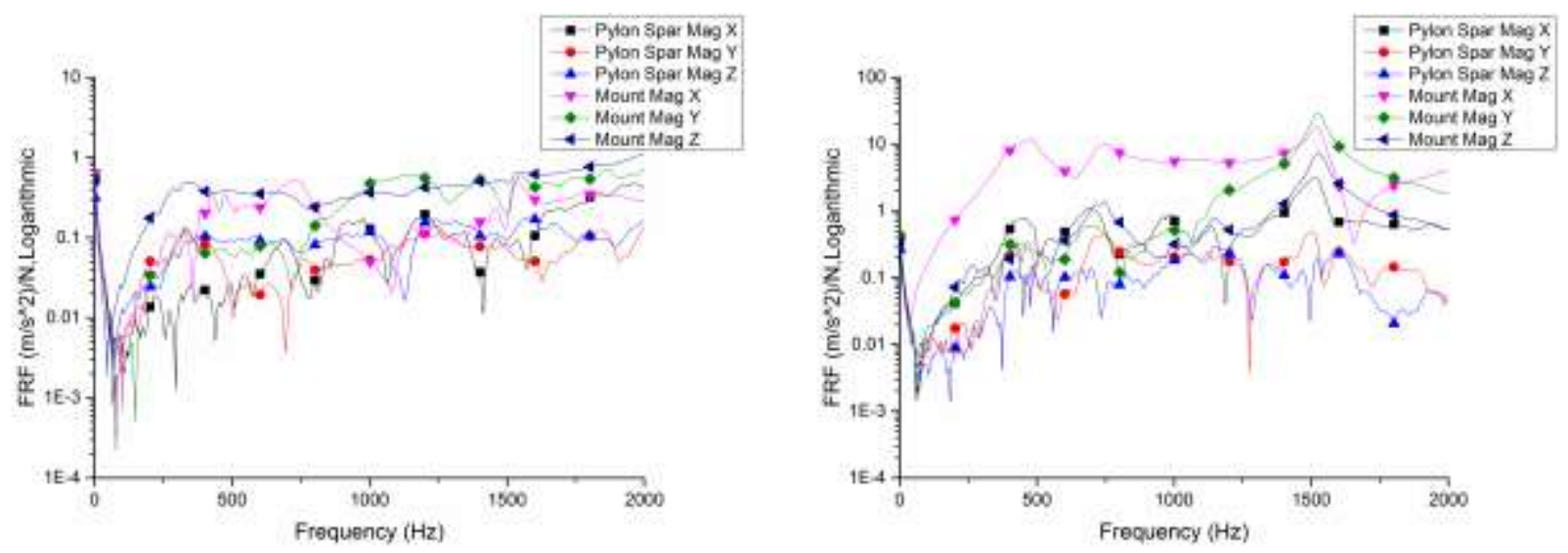

Fig. 4: FRFs of lower excitation point at $\mathrm{Z}$ direction.

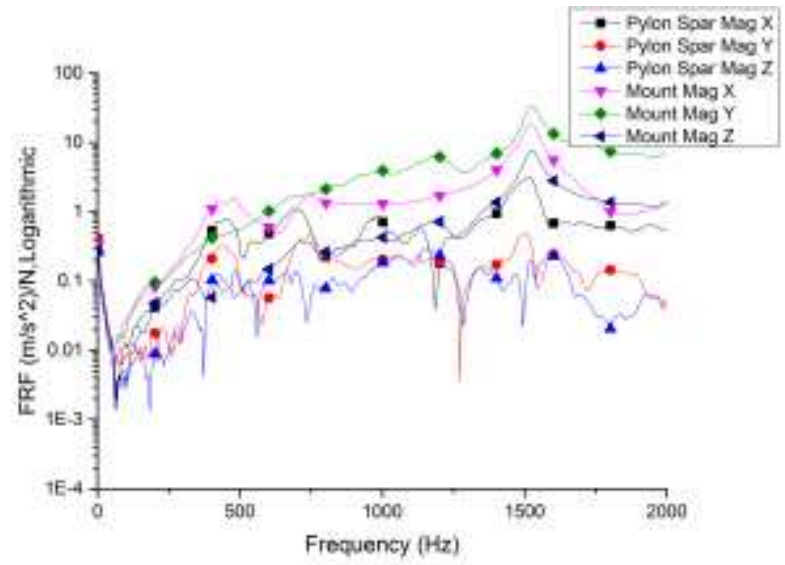

Fig. 5: FRFs of upper excitation point at $\mathrm{X}$ direction.

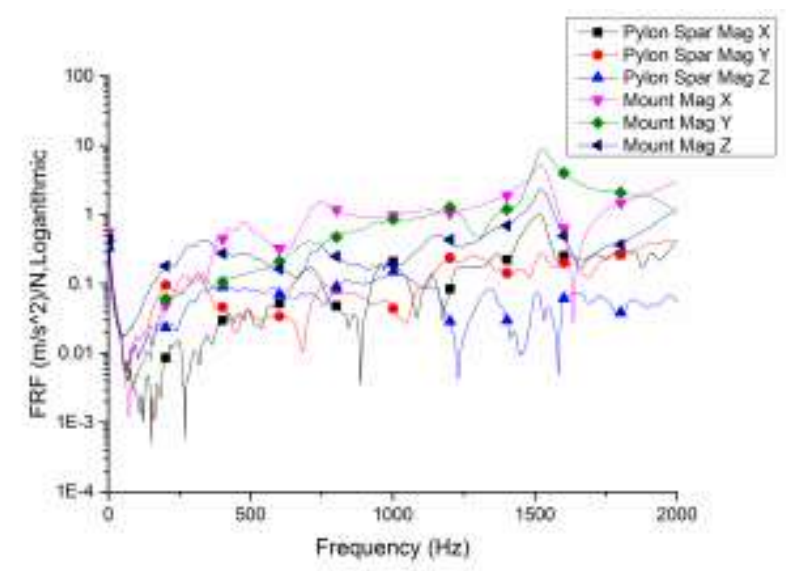

Fig. 6: FRFs of upper excitation point at Y direction. Fig. 7: FRFs of upper excitation point at Z direction.

\section{Acceleration Measurement of Response Locations in Cruise Condition}

In order to back-calculate the dynamic forces on the excitation points of engine mount in cruise condition, the vibration acceleration of the same response locations should be measured when the aircraft is in flight. Fig. 8 and Fig. 9 shown the actual tri-axial accelerometers installation on the mount and pylon.

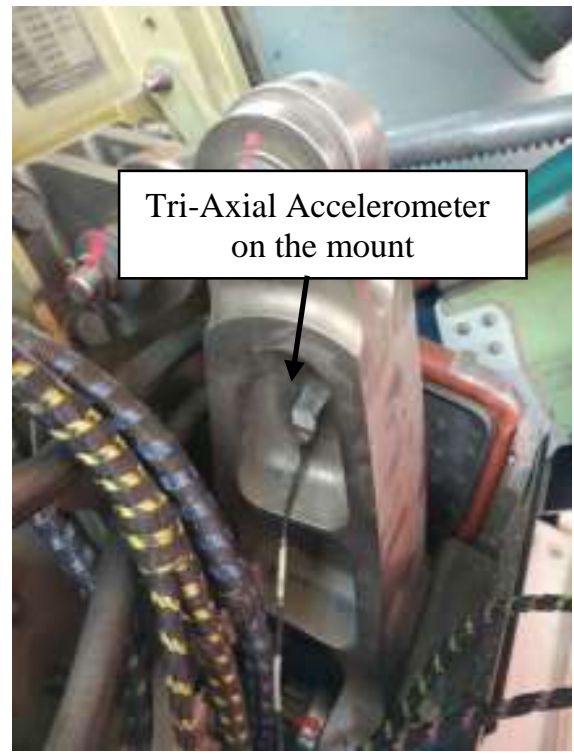

Fig. 8: Tri-axial accelerometer on the mount.

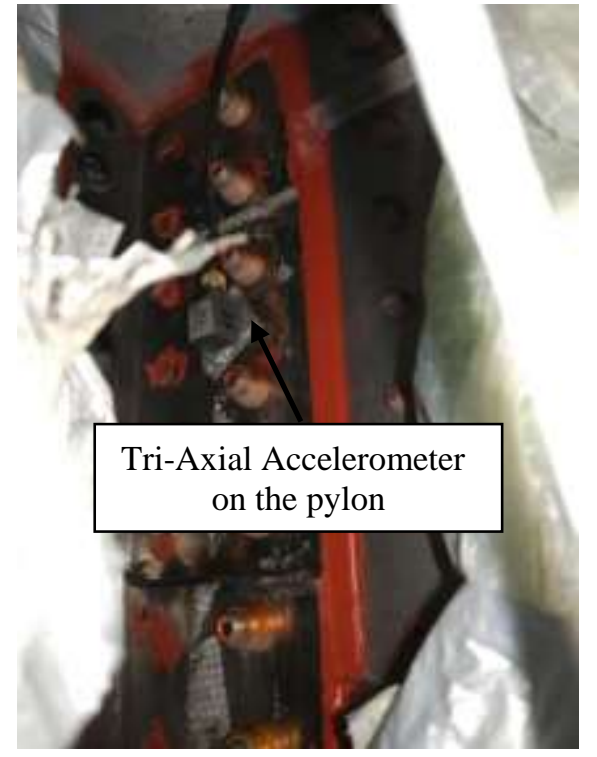

Fig. 9: Tri-axial accelerometer on the pylon.

The flight parameters of aircraft in cruise condition were that the altitude was $35,000 \mathrm{ft}$ and the speed was 0.78 mach. The acceleration vibration responses were measured by data acquisition and accelerometers, and the acceleration vibration spectrums of the two locations were shown in Fig. 10 and Fig. 11. 


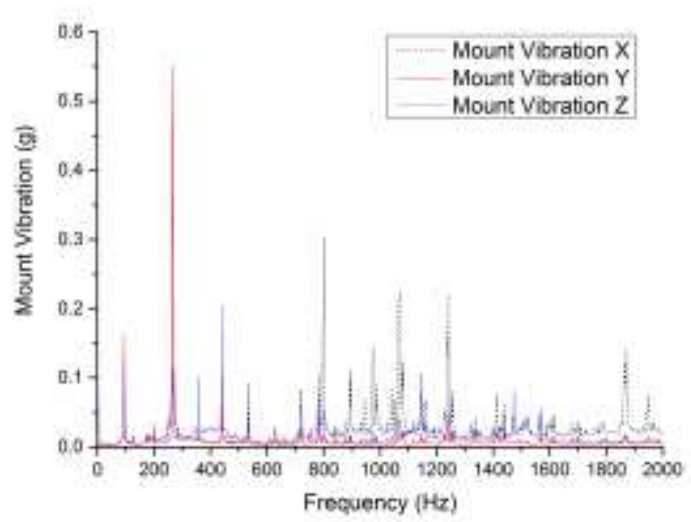

Fig. 10: Acceleration spectrum on the mount.

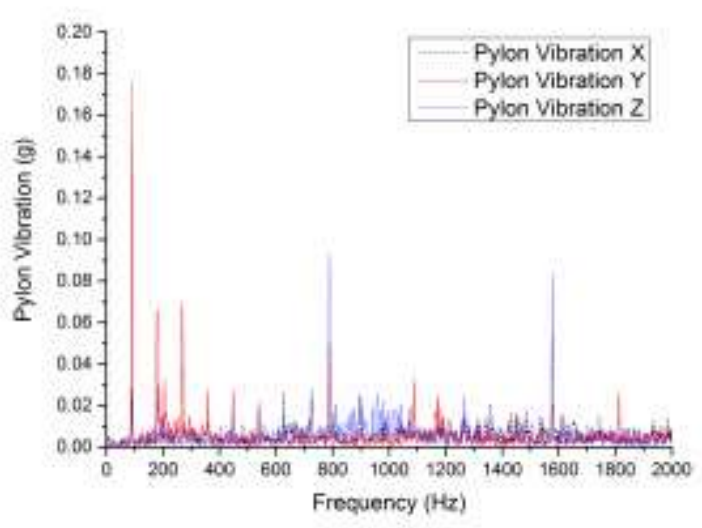

Fig. 11: Acceleration spectrum on the pylon.

\section{Engine Mount Excitation Points Dynamic Forces Identification}

Based on the theory of least-squares scheme in section 2, the engine mount excitation points dynamic forces can be back-calculated with the FRF matrices and measured acceleration responses. In the section 3, two groups of FRF matrices were measured, therefore, the two groups of FRFs matrices would be used to identify the dynamic forces on the engine mount excitation points. Fig. 12 and Fig. 13 show the identified forces by the FRF matrices between lower/upper excitation points and acceleration response point at the pylon with measured acceleration spectrum.

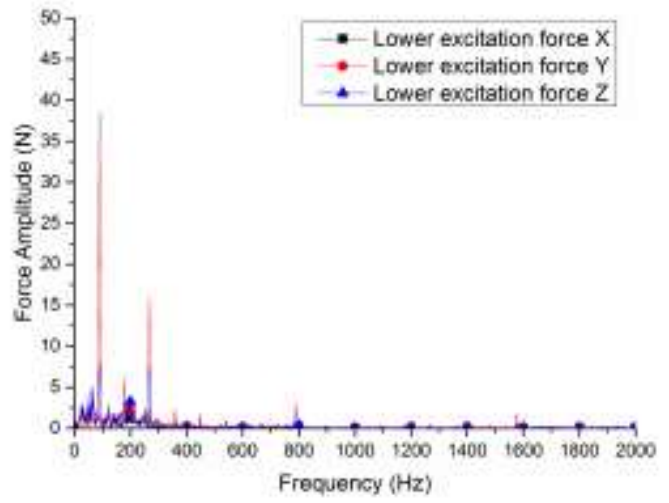

Fig. 12: Forces at the lower point (the 1st matrices). Fig. 13: Forces at the upper point (the 1st matrices).

Fig. 14 and Fig. 15 show the identified forces by the FRF matrices between lower/upper excitation points and acceleration response point at the mount with measured acceleration spectrum.

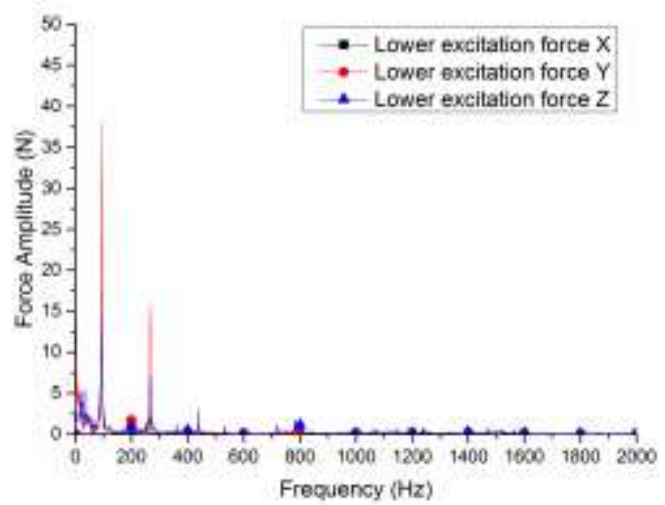

Fig. 14: Forces at the lower point (the 2nd matrices). Fig. 15: Forces at the upper point (the 2nd matrices).

From Fig. 12 to Fig. 15, it is clear that the two groups of FRF matrices both capture the dynamic forces caused by engine low pressure shaft vibration near $90 \mathrm{~Hz}$ and engine high pressure shaft vibration near 
$260 \mathrm{~Hz}$, but the amplitude and the dynamic forces value at other frequencies also exist differences. In order to compare the identified forces from the two groups of FRF matrices, the norm condition numbers are compared with each other as Fig. 16.

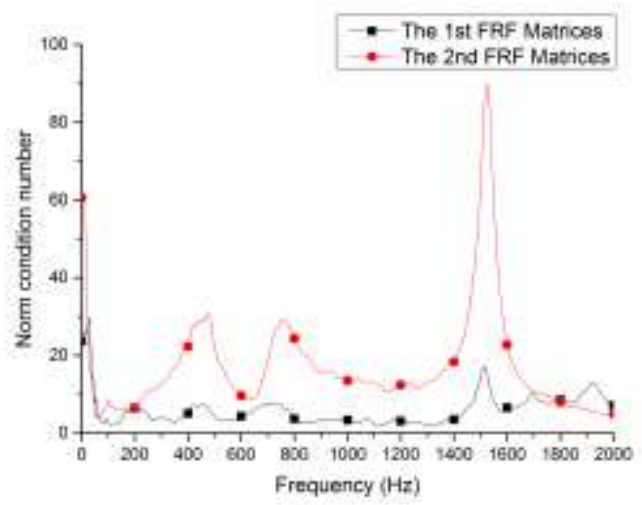

Fig. 16: Comparison of norm condition numbers.

The Fig. 16 shows that norm condition numbers of the two inverse calculated process are below 100, and they both meet the requirement for the use of engineering. But, it is very obvious that the 1st FRF matrices which belongs to the lower/upper excitation points and acceleration response point at the pylon owns a lower norm condition number, it means that the engine mount dynamic forces identified by this FRF matrices are more accurate than the other one and can be treat as the 'exact' value. In this paper, root mean square (RMS) values of these identified forces are used to evaluate the relative errors between them and the errors are shown in Table 1

Table 1: Relative error for the identified dynamic forces of the $1^{\text {st }}$ and $2^{\text {nd }}$ FRF matrices

\begin{tabular}{|c|c|c|c|c|c|c|}
\hline \multirow{2}{*}{$\begin{array}{ll}\text { Forces } & \text { Locations } \\
\end{array}$} & \multicolumn{3}{|c|}{ Lower excitation point on the mount } & \multicolumn{3}{|c|}{ Upper excitation point on the mount } \\
\hline & $\mathrm{X}(\mathrm{N})$ & $\mathrm{Y}(\mathrm{N})$ & $\mathrm{Z}(\mathrm{N})$ & $\mathrm{X}(\mathrm{N})$ & $\mathrm{Y}(\mathrm{N})$ & $\mathrm{Z}(\mathrm{N})$ \\
\hline $\begin{array}{l}\text { Identified Forces RMS } \\
\text { (The } 1^{\text {st }} \text { FRF Matrices) }\end{array}$ & 50.76 & 52.96 & 24.01 & 26.80 & 100.02 & 22.08 \\
\hline $\begin{array}{l}\text { Identified Forces RMS } \\
\text { (The } 2^{\text {nd }} \text { FRF Matrices) }\end{array}$ & 38.84 & 50.61 & 28.10 & 16.84 & 80.38 & 21.76 \\
\hline Relative Errors (\%) & $23.48 \%$ & $4.44 \%$ & $17.03 \%$ & $37.16 \%$ & $13.32 \%$ & $14.49 \%$ \\
\hline
\end{tabular}

Comparing the relative errors shown in Table 1, it can be found that the identified force of the upper excitation point at $\mathrm{X}$ direction has an obvious difference, but for the identified forces at $\mathrm{Y}$ direction, the difference is very small. These identified forces have the similar magnitude order, and they both can be acceptable in engineering. The reason why these differences exist may be the errors belong to FRF matrices or acceleration response measurement in flight condition.

\section{Conclusion}

Aircraft engine mount is a very important structure to attach the engine to the airframe. In order to predict the structural dynamic behaviour and cabin noise caused by the engine vibration, the dynamic forces on the engine mount should be known, but unfortunately, these dynamic forces can't be measured directly, so an effective approach to back-calculate these dynamic forces are required. In this paper, the theory for the dynamic forces identification based on least-squares scheme was introduced in details. The measured response signals and the FRF matrices between the excitation locations and the same response locations were required before the inversion process. For the engineering application in this paper, the FRF matrices between the engine mount lower/upper excitation points and acceleration response point at the pylon, and the FRF matrices between lower/upper excitation points and acceleration response point at the mount were measured when the aircraft parked in hangar. Then, the acceleration signals of the mount response locations and pylons locations were measured when aircraft was in cruise condition. Based on these data and the least- 
squares scheme, the inverse calculation successfully identified the dynamic forces on the engine mount at the lower and upper excitation locations. Norm condition numbers were used to evaluate the inverse calculation process, furthermore, the relative errors between these identified forces were calculated. Based on above work, the conclusion can be obtained as follows

- The least-squares scheme for the dynamic forces identification is implementable and the engine mount dynamic forces in flight condition have been identified successfully with this method.

- The norm condition number is a criterion for the inverse calculation in mathematics and it is also can be used as an indicator to evaluate the identified forces process.

- Due to the absolutely 'exact' dynamic forces in flight condition can't be acquired, the relative errors of identified dynamic forces with different two groups of FRF matrices were calculated. The results show that only the forces of the upper excitation point at $\mathrm{X}$ direction has an obvious difference, but the difference of the absolute values can be acceptable in engineering.

- This paper illustrates all detailed process of the dynamic forces identification, with the same process, other similar engineering problems can be resolved by this method.

\section{Acknowledgements}

At the end of this paper, I'd like to express my sincere thanks to my colleagues in Shanghai Aircraft Design and Research Institute. With the help of Mr.Zixin Feng and Miss Chenxi Li, the theory of the dynamic forces identifications was collected from different kinds of papers. Mr. Feng Han and Mr. Huayong Zhao helped me about the FRF test on the aircraft so that I could get the experimental test results easily. Thank all of you.

\section{References}

[1] Tomoaki Kodama, Yasuhiro Honda. A study on the Modeling and Dynamic Characteristics of the Viscous Damper Silicone Fluid by Using Vibration Control of Engine Crakshaft System. International Journal of Mechanical Engineering and Robotics Research. 2018, 7(3):273-278.

[2] K.Tulegenova, G.Abdraimova, B.kyrykbaev, A.Zhauyt, A.Alimbetov and M.Nurbakyt. Dynamic Analysis of the Press Automation. International Journal of Mechanical Engineering and Robotics Research. 2019, 8(1):41-45.

[3] Yi, Liu, W.Steve Shepard Jr. Dynamic force identification based on enhanced least squares and total least-squares schemes in the frequency domain. Journal of Sound and Vibration. 2004, 282(2005):37-60.

[4] Xun Xu, Jinping Ou, Force identification of dynamic systems using virtual work principle. Journal of Sound and Vibration. 2015, 337(2015):71-94.

[5] Du Jianbin. Structural optimization and its application in vibration and acoustic design. Beijing: Tsinghua University Press, 2015. (in Chinese)

[6] W.G.Bartlett Jr., F.D. Flannelly, Modal verification of force determination for the measuring vibratory loads, Journal of American Helicopter Society. 1979, 24(2):10-18.

[7] N. Okubo, S. Tanabe, T.Tatsuno, Identification of forces generated by a machine under operating condition, The International Modal Analysis Conference, 1985.

[8] J.A. Fabunmi, Modal constraints on structural dynamic force determination, Journal of the American Helicopter Society, 1985, 30(4):48-54.

[9] J.A. Fabunmi, Effects of structural modes on vibratory force determination by the pseudo inverse technique, AIAA Journal, 1986, 24(3):504-509.

[10] Zhanglei, Cao Yueyun, Yang Zichun, He Yuanan. Load Identification using CG-TLS regularization algorithm. Journal of Vibration and Shock, 2014,33(9):159-164. (in Chinese).

[11] C.R. Vogel, Computational Methods for Inverse Problems, SIAM, PHILADELPHIA, 2002.

[12] Lu Qiuhai, Li Lianyou, Xiang Lvkai, Wang Bo. Operational modal parameter identification of structures for nonstationary ambient excitation. Journal of Tsinghua University (Science and Technology), 2013, 53(3):389-393. (in Chinese). 
[13] S.E.S. Karlsson, Identification of external structure loads from measured harmonic responses, Journal of Sound and Vibration. 1996, 196(1):59-74.

[14] Sreejith S Nair. Experimental Investigation of Multipass Tig Welding Using Response Surface Methodology. International Journal of Mechanical Engineering and Robotics Research.2013,2(3):242-254. 